\title{
Perfil Epidemiológico De Lesões De Violência Física Contra Mulheres: Estudo Transversal
}

\section{Epidemiological Profile Of Physical Violence Injuries Against Women: A Transversal Study}

\author{
Andressa da Silva Arduim, Eduardo Dickie de Castilhos, Lenise Menezes \\ Seerig, Tania Izabel Bighetti
}

\section{RESUMO}

Descrever o perfil epidemiológico de mulheres que sofreram violência física, bem como caracterizar suas lesões físicas. Estudo transversal a partir de dados secundários obtidos de laudos periciais da 3a Coordenadoria Médico Legal de Pelotas/RS, de janeiro/2014 a dezembro/2014. Foram analisados 1.330 laudos, sendo 1.164 de mulheres residentes em Pelotas. A cada 1.000 mulheres de 12-44 anos, 10 foram vítimas de violência; $60,6 \%$ mulheres de $20-44$ anos, a maioria solteira $(74,6 \%)$ e de cor branca $(85,2 \%)$. O período de primavera/verão foi o de maior prevalência, correspondendo a $57 \%$ dos laudos. As regiões do corpo mais atingidas foram face e cabeça $(36,4 \%)$ e membros superiores $(45,8 \%)$. 0 delineamento do perfil epidemiológico das mulheres vitimadas e sua correta notificação permitem uma melhor abordagem da problemática violência contra mulher, destacando a importância do cirurgião-dentista no atendimento integral e humanizado. Fundamenta-se assim a abordagem do tema violência contra mulher nos cursos de graduação de Odontologia.

Descritores: Epidemiologia. Violência contra a mulher. Políticas públicas.

Como citar este artigo:

Arduim AS, Castilhos ED, Seerig LM, Bighetti TI. Perfil epidemiológico de lesões de violência física em mulheres: estudo transversal. Revista Saúde (Sta. Maria). 2018; 44(1): $1-9$

Autor correspondente:

Andressa da Silva

Email: andressa.arduin@gmail.com

Telefone: (53) 98125-8862

Endereço: Praça da Liberdade,

258, areal

Pelotas-RS

Data de Submissão: 08/06/2017

Data de aceite: $08 / 02 / 2018$

Conflito de Interesse: Não há conflito de interesse.

\section{ABSTRACT}

To describe the epidemiological profile of women who have suffered violence and to characterize their physical injuries. A transversal study was conducted based on secondary data obtained from expert reports in the 3rd Regional Coordination of Health of Pelotas, from January/2014 to December/2014. Of 1,330 reports analyzed, 1,164 were related to women living in Pelotas. In one thousand women aged 12-44 years, around 10 were victims of violence during the study period; $60.6 \%$ women aged $20-44$ years, most single $(74.6 \%)$ and white $(85.2 \%)$. The spring/summer period was the most prevalent indicating $57 \%$ of reports. Face and head $(36.4 \%)$ and upper limbs $(45.8 \%)$ were more frequently hitted. The blunt instrument was present in $88.9 \%$ of the cases and $8.4 \%$ were without information. The epidemiological profile of victimized women and their proper notification allow a better approach to domestic violence, highlighting the importance of the dentist in full and humanized care. It is based on this the approach violence against women in the undergraduate courses in Dentistry.

Descriptors: Epidemiology. Violence Against Women. Public Policies. 


\section{Introdução}

A violência doméstica tem como pressuposto que ocorra no âmbito da unidade doméstica, compreendida como o espaço de convívio permanente de pessoas, com ou sem vínculo familiar, inclusive as esporadicamente agregadas ${ }^{1}$. Adicionalmente a violência contra a mulher é definida como qualquer ato de violência motivada pelas expressões de desigualdades baseadas na condição de sexo, a qual começa no universo familiar, onde as relações de gênero se constituem no protótipo de relações hierárquicas que resulta ou pode resultar, em dano ou sofrimento de natureza física, sexual ou psicológica; quer se produzam na vida pública ou privada ${ }^{2}$. Representa um grande desafio no âmbito social, político e cultural. É uma questão de saúde pública, já que com uma taxa de 4,8 homicídios em 100 mil mulheres, o Brasil ocupa a quinta posição no contexto dos 84 países do mundo com dados homogêneos entre 2006 e $2013^{3}$.

Apenas uma em cada dez mulheres em situação de violência que procuram atendimento médico é oficialmente reconhecida pelos profissionais de saúde como mulher espancada. Na maioria dos casos, a agressão é negada ou sua importância é diminuída. As respostas médicas às mulheres espancadas tendem a se limitar ao tratamento das lesões físicas causadas pelo espancamento4.

As causas dessa "não percepção" dos casos de violência devem-se provavelmente a várias circunstâncias como: despreparo dos profissionais da saúde, desinteresse, falta de tempo para escutar o paciente durante os atendimentos, ater-se somente às lesões físicas, e a uma cultura de que "em briga de marido e mulher, ninguém mete a colher"5.

Logo, na rotina odontológica o profissional pode se deparar com traumatismos diversos nas estruturas do complexo bucomaxilofacial6, uma vez que na sua área de domínio, cabeça e pescoço frequentemente são alvos de lesões de violência. A conduta do odontólogo frente a esta situação deve ser de acolhimento e empoderamento da vítima para que a mesma sinta-se amparada e procure seus direitos. Além disso, está presente a responsabilidade ético-legal de denunciar às autoridades competentes os casos de violência que forem identificados durante a prática clínica, por meio de uma notificação compulsória7,8, exercendo o seu dever como ator social de garantia de direito e proteção social às vitimas. Este instrumento de registro possibilita a visibilidade deste fenômeno. Também deverá registrar adequadamente em prontuário bem estruturado, arquivando devidamente toda a documentação produzida em função dos atendimentos clínicos e atentar para o sigilo profissional9.

Norteado pelo "Plano Nacional de Políticas para Mulheres"10 e visando o enfrentamento da violência está contemplado como objetivo geral a redução dos índices de todas as formas de violência contra a mulher. Considerando que o número de vítimas e magnitude das sequelas orgânicas e emocionais que produz, adquiriu um caráter endêmico e se converteu num problema de saúde pública, como reconhecido pela Organização Pan-Americana de Saúde11. Fica evidente o compromisso que os profissionais de saúde têm em detectar e identificar os casos de violência e suas vítimas já que são eles que prestam os primeiros atendimentos a elas.

O objetivo deste estudo é descrever o perfil epidemiológico de mulheres que sofreram de violência física e caracterizar as lesões geradas pelas agressões.

\section{Metodologia}

O projeto foi aprovado pelo Comitê de Ética em Pesquisa da Faculdade de Odontologia da Universidade Federal de Pelotas (protocolo $n^{0}$ 14/2014). Uma autorização foi solicitada ao Departamento Médico Legal (DML) de Pelotas-RS e um relatório com os principais resultados foi enviado ao serviço. Justifica-se a dispensa do Termo de Consentimento Livre e Esclarecido para a coleta dos dados em questão, pois a análise estatística dos dados foi baseada em princípios epidemiológicos, não avaliando dados individuais. A análise do banco de dados do DML teve caráter exclusivamente estatístico, assim ficou resguardada a identidade dos indivíduos.

Realizou-se um estudo do tipo censo, descritivo, transversal, a partir de dados secundários. Foi conduzido no DML de Pelotas, que é referência para situações de violência e baseou-se em laudos periciais de mulheres amparadas pelo serviço. $O$ período de coleta dos dados deu-se de janeiro de 2014 a dezembro de 2014. Realizou-se um estudo piloto durante o mês de janeiro de 2014 para teste da coleta de dados e adaptação da planilha. Uma única pesquisadora realizou a extração dos dados.

Durante o processo de seleção de laudos relevantes à pesquisa foram excluídos os laudos referentes a acidentes de trânsito, acidentes com animais, acidentes de queda da própria altura, laudos sendo a vítima do sexo masculino e 
conjunção carnal, devido a não permissão de acesso a este tipo de laudo.

As variáveis de estudo relacionadas à caracterização sociodemográfica das mulheres foram: idade apresentandose nas faixas etárias: 0 a 11 anos; 12 a 19 anos; de 20 a 34 anos; 35 a 44 anos; 45 a 59 anos e mais que 60 anos 12 . Em relação à cor da pele classificou-se em branca, preta e parda. De acordo com o estado civil apresentaram-se as seguintes qualificações: solteira, união estável e divorciada/viúva. Quanto à localização de moradia baseou-se na legislação que criou os distritos sanitários13, ficando assim classificados para o município de Pelotas-RS: Três Vendas, Centro, Fragata, Areal/Laranjal e Colônia. A partir de uma classificação inicial pela Classificação Brasileira de Ocupações14 foram criadas as seguintes categorias: (1) militares, nível superior, nível médio e indústria e manutenção; (2) trabalhadores rurais, estudantes, pensionistas e do lar; e (9) sem informação.

As variáveis relacionadas à caracterização das lesões de violência foram: estação do ano; (primavera, verão, outono e inverno); local da lesão (face e cabeça; membros superiores; tronco; membros inferiores; corpo inteiro) codificado como sim ou não; instrumento/meio que produziu a lesão (veneno; fogo; explosivo; asfixia ou tortura ou por outro meio insidioso ou cruel) codificado como sim ou não; gravidade da lesão codificada como sim ou não, e instrumento ou meio que produziu a ofensa (cortante; cortante/contundente; contundente; outro).

Os dados foram digitados em uma planilha do programa Microsoft Office Excel versão 2010, transferidos para 0 programa Stata versão 12, e analisados de forma descritiva.

Para as mulheres provenientes de Pelotas, foi calculada a prevalência por faixa etária a partir de estimativas populacionais do ano de $2014^{12}$.

\section{RESULTADOS:}

Foram analisados 1.330 laudos de mulheres assistidas no DML, no período de janeiro de 2014 a dezembro de 2015. Deste total, 1.164 se referiam a mulheres residentes em Pelotas-RS. Os aspectos relativos à caracterização sociodemográfica destas mulheres estão apresentados na Tabela 1.

Tabela 1 - Caracterização sociodemográfica de mulheres vítimas de violência física. Pelotas, RS, 2014-2015.

\begin{tabular}{|c|c|c|c|c|c|}
\hline \multirow[t]{2}{*}{ Variável } & \multicolumn{2}{|c|}{ Episódios de violência } & \multicolumn{2}{|c|}{ População } & \multirow{2}{*}{$\begin{array}{l}\text { Casos/ } \\
1.000 \text { habitantes }\end{array}$} \\
\hline & No & $\%$ & $\mathrm{~N}$ & $\%$ & \\
\hline \multicolumn{6}{|l|}{ Faixa etária15 } \\
\hline 0 a 11 anos & 15 & 1,3 & 25.122 & 13,8 & 0,6 \\
\hline 12 a 19 anos & 221 & 19,0 & 19.345 & 10,7 & 11,4 \\
\hline 20 a 34 anos & 341 & 29,3 & 32.531 & 17,9 & 10,5 \\
\hline 35 a 44 anos & 365 & 31,3 & 37.307 & 20,6 & 9,8 \\
\hline 45 a 59 anos & 163 & 14,0 & 36.007 & 19,8 & 4,5 \\
\hline $60 \mathrm{e}+$ anos & 59 & 5,1 & 31.310 & 17,2 & 1,9 \\
\hline \multicolumn{6}{|l|}{ Cor da pele } \\
\hline Branca & 992 & 85,2 & & & \\
\hline Preta & 129 & 11,1 & & & \\
\hline Parda & 42 & 3,6 & & & \\
\hline Sem informação & 1 & 0,1 & & & \\
\hline \multicolumn{6}{|l|}{ Estado civil } \\
\hline Solteira & 868 & 74,6 & & & \\
\hline União estável & 178 & 15,3 & & & \\
\hline Divorciada/viúva & 116 & 10,0 & & & \\
\hline Sem informação & 2 & 0,1 & & & \\
\hline \multicolumn{6}{|l|}{ Distrito14 } \\
\hline Três Vendas & 289 & 24,8 & & & \\
\hline Centro & 250 & 21,5 & & & \\
\hline Fragata & 250 & 21,5 & & & \\
\hline
\end{tabular}




\begin{tabular}{lrr} 
Areal/Laranjal & 274 & 23,5 \\
Colônias & 12 & 1,0 \\
Sem informação & 89 & 7,7 \\
& & \\
Ocupação & & \\
Militares/nível superior/nivel médio/indústria e manutenção & 519 & 44,6 \\
Rurais/estudantes/pensionistas/do lar & 617 & 53,0 \\
Sem informação & 28 & 2,4 \\
\hline \multirow{2}{*}{ Total } & \multirow{2}{*}{1.164} & 100,0 \\
\hline
\end{tabular}

Observou-se que, no período estudado, a cada 1.000 mulheres de 12 a 44 anos de idade, em torno de 10 foram vítimas de violência no município de Pelotas-RS. A quantificação de agressões de acordo com a faixa etária é demostrada no Gráfico 1.

Gráfico 1 - Número de episódios de violência física contra a mulher de acordo com a faixa etária. Pelotas, RS, 2014-2015.

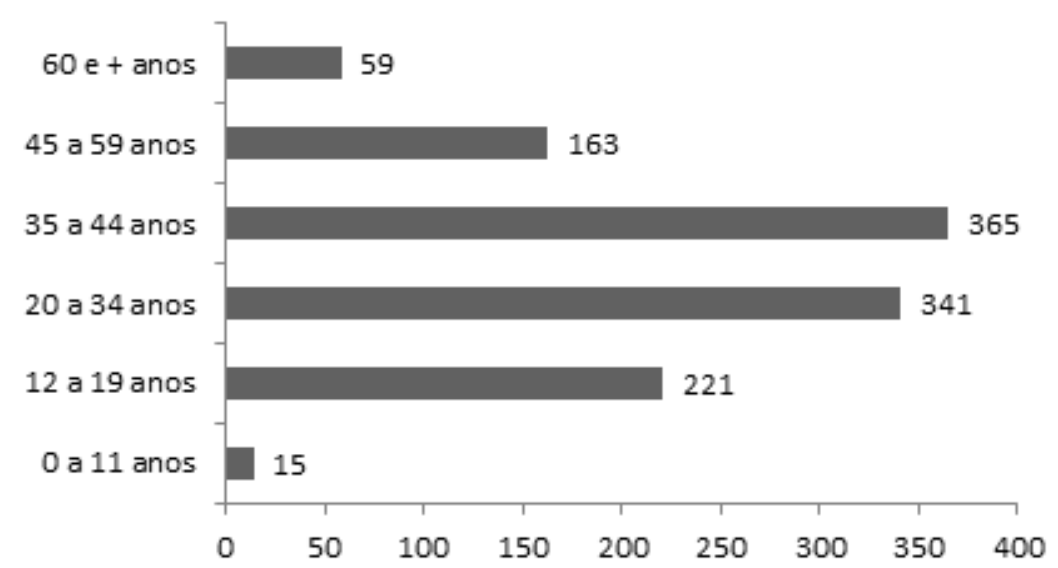

Avaliando-se os dados referentes ao perfil das vítimas notou-se que mulheres de todas as faixas etárias estavam envolvidas, com maior proporção $(60,6 \%)$ mulheres de 20 a 44 anos, a maioria solteira $(74,6 \%)$ e de cor de pele branca $(85,2 \%)$. A distribuição entre os quatro distritos de residência foi semelhante e foi observada pequena prevalência mulheres da zona rural (1\%). As ocupações mais prevalentes no estudo foram as que pertenciam à categoria que agrupou trabalhadores rurais, do lar, estudantes e pensionistas perfazendo $53 \%$ dos laudos, seguida pela que agrupou militares, nível superior, nível médio e trabalhadores de indústria e manutenção (44,6\%).

A Tabela 2 apresenta a caracterização das lesões de física observada nos laudos.

Tabela 2 - Caracterização das lesões de violência física em mulheres. Pelotas, RS, 2014-2015.

\begin{tabular}{lrr}
\hline Variável & $\mathrm{n}^{0}$ & $\%$ \\
\hline Estação do ano & & \\
Primavera & 290 & 24,9 \\
Verão & 379 & 32,6 \\
Outono & 260 & 22,3 \\
Inverno & 235 & 20,2 \\
& & \\
Local da lesão & & \\
Face e cabeça & & \\
Não & 648 & 55,7 \\
Sim & 424 & 36,4 \\
Sem informação & 92 & 7,9 \\
& & \\
Membros superiores & & \\
Não & 539 & 46,3 \\
Sim & 533 & 45,8
\end{tabular}




\begin{tabular}{lrrr} 
Sem informação & & 92 & 7,9 \\
Tronco & & \\
Não $\quad 883$ & 75,9 & \\
Sim & 189 & 16,2 \\
Sem informação & 92 & 7,9 \\
& & \\
Membros inferiores & & \\
Não & 735 & 62,8 \\
Sim & 341 & 29,3 \\
Sem informação & 92 & 7,9 \\
Corpo inteiro & & \\
Não & 1.054 & 90,6 \\
Sim & 18 & 1,5 \\
Sem informação & 92 & 7,9 \\
\hline Total & 1.164 & 100,0 \\
\hline
\end{tabular}

O verão foi identificado como a estação do ano de maior prevalência de violência física no município correspondendo a $32,6 \%$ dos laudos. Das regiões do corpo que foram atingidas, face e cabeça $(36,4 \%)$ e membros superiores $(45,8 \%)$ foram as mais prevalentes.

$O$ instrumento de ofensa de maior prevalência foi o contundente identificado em $88,9 \%$ dos casos. Em dois casos a lesão foi produzida por meio insidioso ou cruel através de veneno, fogo, explosivo, asfixia ou tortura. No que diz respeito a gravidade da lesão codificada como sim ou não, foram identificados dois casos com resposta positiva.

\section{Discussão}

A principal característica deste estudo foi estimar a prevalência de violência física em mulheres do município de Pelotas-RS no período de janeiro de 2014 e dezembro de 2014. Observou-se que, a cada 1.000 mulheres de 12 a 44 anos de idade, em torno de 10 foram vítimas de violência no município de Pelotas-RS no período estudado.

Um obstáculo evidente no transcorrer do estudo foi a classificação do tipo de violência identificada, dificultando a classificação em violência contra mulher ou violência doméstica, uma vez que não haviam informações a respeito do local no qual ocorreram as agressões, dado primordial para tal caracterização, sendo assim utilizou-se o termo "violência contra a mulher" para caracterizar a pesquisa.

Alguns estudos16,17 que abordam a "violência contra a mulher" denominam como "violência doméstica". Porém, observa-se que esta nomenclatura é usada de forma genérica, sem haver a preocupação de caracterizar o ambiente onde ocorreu a agressão e tampouco o gênero e suas consequências e sim a questão do sexo.

A violência contra a mulher é um problema de alta relevância pelo seu elevado número de casos e ao mesmo tempo pela sua invisibilidade. O fato de se observar neste estudo que mulheres de todas as faixas etárias foram vitimadas, reforça a necessidade de se discutir este evento como um problema de saúde pública. Quadros orgânicos podem ser resultantes das lesões provocadas, como distúrbios de sono, síndrome de dor crônica, distúrbios gastrintestinais, cansaço, sofrimento psíquico, invalidez, bem como maior uso de medicamentos ansiolíticos e antidepressivos 18, que vão exigir ações preventivas e assistenciais dos mais diversos níveis de complexidade.

Uma das características do estudo diz respeito ao fato de os dados serem de origem secundária, desta maneira, laudos preenchidos de maneira incompleta, ou até mesmo ausência de preenchimento, podem conduzir o estudo a conclusões equivocadas. Laudos com situações equivalentes, porém com constatações divergentes sugerem falta de padronização e subjetividade nas avaliações, assim podem conferir resultados não fidedignos a situação.

Os principais achados apontaram que mulheres em sua maioria solteiras e de cor branca, sendo mais da metade na faixa etária de 20 a 44 anos, tendem a serem vítimas da violência, e que um terço das observações de violência física se deu no verão. Talvez, o motivador seja o fato da exposição do corpo ser maior durante esta estação, mais uma 
vez evidenciando a desigualdade entre os gêneros, marcada pelas evidências machistas na sociedade. Além disso, as temperaturas elevadas sugestionam o consumo de álcool e uma vida social mais ativa.

O fato de a faixa etária de 20 a 44 anos de idade representar em torno de $61 \%$ dos casos de violência pode ser explicado por ser o período de maior atividade sexual das mulheres19. Outras faixas etárias foram apontadas como de maior prevalência para a violência contra a mulher, como a de 0 a 15 anos20, mas isto pode estar relacionado ao fato de os dados serem inquéritos policiais e do núcleo de vigilância de violência de Porto Alegre. Essa divergência sugere que a mulher adulta tem o livre arbítrio de representar ou não contra seus agressores, já a criança e o adolescente ficam sujeitos à obrigatoriedade que a autoridade policial tem de instaurar inquérito uma vez a suspeita de maus tratos.

Segundo o Instituto Brasileiro de Geografia e Estatística (IBGE)21 em 2010, 53\% da população do município de Pelotas era composta por mulheres, e destas, $5,7 \%$ se autodeclararam como pretas e $42,7 \%$ como brancas. A partir desta informação compreende-se o resultado de que a maioria das mulheres presentes na pesquisa seja branca, refletindo a maioria da população em geral.

Houve uma homogeneidade na distribuição das mulheres agredidas em relação ao bairro de residência em Pelotas. Isto pode estar relacionado ao agrupamento de bairros com diferentes características socioeconômicas dentro de um mesmo distrito. $O$ fato de o serviço ser uma referência para este tipo de laudo pericial pode justificar casos relativos a outros municípios.

As ocupações classificadas na categoria que envolveu trabalhadores rurais, do lar, pensionistas e estudantes em $53 \%$ das mulheres podem estar relacionadas à dependência e submissão ao agressor, porém não há evidências de tal afirmação sugerindo assim maior aprofundamento do assunto.

Verificou-se que quando houve lesão de cabeça e pescoço, esta representou um terço das ocorrências. Estudos realizados em Porto Alegre/RS e Ceará,20,22 também observaram esta parte do corpo com prevalências de 10,4\% e $28,3 \%$ respectivamente. A região ocupada pela cabeça apresenta grande vulnerabilidade sendo assim compatível com a região de maior incidência das lesões. A justificativa para a região de face ser a de preferência do agressor se dá pelo caráter simbólico de humilhação expressado quando o rosto da mulher é atingido. A intenção do agressor é tornar visível a lesão e com isso prejudicar um atributo muito valorizado socialmente que é a beleza feminina, além de produzir sentimentos de auto depreciação e vergonha gerando acentuado sofrimento psíquico23.

O comprometimento do complexo maxilomandibular nos crimes de lesão corporal em mulheres24 reforça a importância da presença do cirurgião-dentista nos serviços de emergência em hospitais. A ausência de um cirurgiãodentista como perito gera laudos pobres em detalhes importantes referentes à região bucomaxilofacial, prejudicando a qualidade das descrições das lesões e suas repercussões no indivíduo. Além disso, os membros superiores, quando afetados, apresentaram metade das ocorrências, e isto pode ser justificado por serem utilizados como defesa no momento da agressão17.

O tipo de agente que o agressor utilizou para produzir a ofensa foi o contundente na maioria dos casos, semelhante a resultados de outros estudos,25,26. De acordo com os dados obtidos detectou-se que em dois casos foi considerado como meio insidioso ou cruel, porém esta avaliação tem algum grau de subjetividade. Constatou-se que em dois casos a lesão foi designada grave o que pode ser justificado pelo fato de essas lesões gerarem detrimento psicossocial e moral em virtude da morfologia aparente20.

Para uma melhor abordagem da problemática violência contra a mulher, ressalta-se a importância do cirurgiãodentista no atendimento integral e humanizado. $O$ correto preenchimento de prontuários e a realização da notificação compulsória aos órgãos competentes servem de subsídios no dimensionamento desse problema de saúde pública, valendo-se de instrumento para planejamento de políticas públicas. Fundamenta-se assim a abordagem do tema violência contra a mulher nos cursos de graduação de Odontologia. Além disto, estudos qualitativos podem vir a fomentar as pesquisas relacionadas à violência contra a mulher, pois permitem maior aprofundamento e detalhamento deste evento de importância em saúde pública.

\section{Conclusão}

O perfil epidemiológico de mulheres que sofreram de violência física na cidade de Pelotas no período de janeiro de 2014 a dezembro de 2014 caracterizou-se pela prevalência de 60,6\% de mulheres de 20 a 44 anos, a maioria solteira 
$74,6 \%$ sendo $85,2 \%$ da cor branca. O período de primavera/verão foi o de maior prevalência correspondendo a $57 \%$ dos laudos, a região de face e cabeça correspondeu a 36,4\% das lesões, o instrumento contundente esteve presente em $89,1 \%$ dos casos, sendo $0,2 \%$ foi realizado por meio insidioso ou cruel, e $0,2 \%$ das lesões classificadas como grave.

O delineamento do perfil epidemiológico das mulheres vitimadas e sua correta notificação permitem uma melhor abordagem da problemática violência contra a mulher, ressaltando a importância do cirurgião-dentista no atendimento integral e humanizado. Fundamenta-se assim a abordagem do tema violência contra a mulher nos cursos de graduação de Odontologia.

\section{Referências}

1. Brasil. Lei no 11.340/2006, de 07 de agosto de 2006. Lei Maria da Penha. Coíbe a violência doméstica e familiar contra a mulher. Brasilia, 2006

2. Bandeira, LM. Violência de gênero: a construção de um campo teórico e de investigação. Sociedade e Estado 2014, 29(2): 449-469.

3. Waiselfisz, JJ. Mapa da Violência 2015. Homicídios de mulheres no Brasil. São Paulo, Instituto Sangari, 2015.

4. Gomes, ND, Garcia, TCS, Conceição, CR, Sampaio, PO, Almeida, VC, Paixão, GPN. Violência conjugal: elementos que favorecem o reconhecimento do agravo. Saúde em Debate 2012; 36(95): 514-522.

5. Garbin, CAS, Rovida, TAS, Costa, AA, Garbin, AJl. Percepção e atitude do cirurgião-dentista frente à violência intrafamiliar em 24 municípios do interior de São Paulo, 2013-2014. Epidemio. Serv. Saúde 2016; 2006; 25(1): 179-186.

6. Pereira, MS, Pereira, MS, Lima, DO, Matos, FTC. Lesões bucofaciais decorrentes de situações de violência doméstica: revisão integrativa de literatura. Vivências 2016, 12(22): 201-211.

7. Brasil. Lei n. ${ }^{\circ}$ 10.778, de 24 de novembro de 2003. Estabelece a notificação compulsória, no território nacional, do caso de violência contra a mulher que for atendida em serviços de saúde públicos ou privados. Brasília; 2003.

8. Garbin, CAS, Dias, IA, Rovida, TAS, Garbin, AJI. Desafios do profissional de saúde na notificação da violência: obrigatoriedade, notificação e encaminhamento. Ciência \& Saúde Coletiva, 2015; 20(6): 1879-1890.

9. Brasil. Conselho Federal de Odontologia. Resolução n. ${ }^{\circ}$ 42, de 20 de maio de 2003. Aprova o Código de Ética Odontológica. Rio de Janeiro; 2003.

10. Brasil. Presidência da República. Secretaria de Políticas para as Mulheres.

Plano Nacional de Políticas para as Mulheres. Brasília: Secretaria de Políticas para as Mulheres, 2013. 114 p.

11. Organização Panamericana da Saúde (OPAS). Health care for women subjected to intimate partner violence or sexual violence. 2014.

12. Brasil. Datasus. Disponivel em: http://www2.datasus.gov.br/DATASUS/index. php?area=0206\&id=6943\&VObj=http://tabnet.datasus.gov.br/cgi/deftohtm.exe?ibge/cnv/popt (acessado em 20 de dezembro de 2017). 
13. Brasil. Lei n. ${ }^{\circ} 6.033$, de 18 de setembro de 2013. Cria distritos sanitários para a atuação junto à secretária municipal de saúde, e dá outras providências. Pelotas; 2013.

14. Brasil. Classificação Brasileira de Ocupações: CBO -2010. 3. ed. Brasília: MTE, SPPE; 2010.

15. Brasil. Datasus. Disponível em: http://www2.datasus.gov.br/DATASUS/index. php?area=0206\&id=6943\&VObj=http://tabnet.datasus.gov.br/cgi/deftohtm.exe?ibge/cnv/popt (acessado em 20 de dezembro de 2017).

16. Andrade, MF, Giuliani, CD, Biffi, EFA. Perfil de mulheres vítimas de violência assistidas no prontosocorro/Hospital de Clínicas-UFU. Revista Fato \& Versões 2011; 3(5): 103-33.

17. Schraiber, LB, D'Oliveira, AFPL, França-Junior, I, Pinho, AA. Violência contra a mulher: estudo em uma unidade de atenção primária à saúde. Revista de Saúde Pública 2002; 36(4): 470-477.

18. Leite, FMC, Silva, ACA, Bravim, LR, Tavares, FL, Primo, CC, Lima,EFA. Mulheres vítimas de violência: percepção, queixas e comportamentos relacionados à saúde. Revista de Enfermagem 2016; 10(6): 4854-4861.

19. Pires, GE, Gomes, EM, Duarte, AD, Macedo, AF. Violência interpessoal em vulneráveis e mulheres: perfil das vítimas e diagnóstico pericial das lesões maxilomandibulares. Oral Scienses 2012; 4(1): 10-17.

20. Figueiredo, MC, Cesar, MO, Silva, JP, Borba, EMB. Prevalência de mulheres vítimas de violência no munícipio de Porto Alegre e a influência de suas variáveis no âmbito odontológico. Rev. Faculdade de Odontologia Passo Fundo 2012; 17(3): 254-260.

21. Instituto Brasileiro de Geografia e Estatística. Censo Demográfico 2010. Disponível na Word Wide Web: http://www.censo2010.ibge.gov.br/ (acessado em 20 de dezembro de 2017).

22. Amaral, LBM, Vasconcelos, TB, Sá, FE, Silva, ASR, Macena, RHM. Violência doméstica e a lei Maria da Penha: perfil das agressões sofridas por mulheres abrigadas em unidade social de proteção. Estudos Feministas 2016; 24(2): 521-540.

23. Dourado, SM, Noronha, CV. Marcas visíveis e invisíveis: danos ao rosto feminino em episódios de violência conjugal. Ciência \& Saúde Coletiva 2016; 20(9): 2911-2920.

24. Pereira, MSP, Pereira, MS, Matos, FTC, Marques, MVF, Sarubbi, GD, Damião, JHF. Oral facial Injuries in women victims of domestic violence: integrative literature review.. Brazilian Journal of Forensic Sciences 2014; 4(1): 1-11.

25. Nascimento, LS, Hage, CA, Nakano, AMS, Azevedo, PSB, Letierre, A. Violência contra a mulher e consequência à saúde bucal. Gênero na Amazônia 2012; 2: 9-2.

26. Mattos, PR, Ribeiro, IS, Camargo, VC. Análise dos casos notificados de violência contra a mulher. Cogitare Enfermagem 2012; 17(4): 738-744. 